\title{
Diversidade Cultural e Tecnologias Sociais: estudos para incentivar a autogestão de comunidades do Brasil e Argentina ${ }^{1}$
}

\author{
Diversidad Cultural y Tecnologías Sociales: estudios para incentivar la \\ autogestión de comunidades de Brasil y Argentina
}

\section{Cultural Diversity and Social Technologies: studies to encourage the self- management of communities in Brazil and Argentina}

\author{
Nadja Maria Mourão \\ Rita de Castro Engler ${ }^{3}$ \\ Ana Célia Carneiro Oliveira ${ }^{4}$
}

\begin{abstract}
Resumo
Em tempos atuais, para combater as constantes crises que assolam as nações em todos os continentes, os padrões pré-estabelecidos não mais atendem as questões vivenciadas pela humanidade. Há uma busca constante por novos modelos de gestão, em todas as áreas e setores que demandam o desenvolvimento. Nesse estudo, apresentam-se dois estudos em comunidades na América Latina (Brasil e Argentina) que constroem experiências participativas para incentivo a autogestão. Acredita-se que as tecnologias sociais, oriundas de ações governamentais e não-governamentais, quase sempre apoiadas em políticas públicas, podem gerar possibilidades empreendedoras sociais, reafirmando suas especificidades identitárias. Pelo método da análise bibliográfica realiza-se uma busca de temáticas que possibilitem uma abrangência de conteúdos e exemplos que atendam aos objetivos da pesquisa. O evento "Encontro dos Povos do Grande Sertão Veredas" é realizado em Chapada Gaúcha, em Minas Gerais/Brasil. evento reúne pessoas de outros municípios, estados e países. Em meio aos festejos, realizam-se a comercialização de produtos locais por meio da moeda social - Veredas. Na Argentina, na periferia da cidade de Buenos Aires, a plataforma "Caminhos da Favela" busca soluções para problemas estruturais das comunidades, que participam da identificação desses problemas e promovem a integração entre os membros. Percebe-se que as tecnologias sociais além de solucionarem problemas pontuais reafirmam a cultura e a identidade das comunidades gestoras. São caminhos percorridos que se transformam em fatores diferenciais para possibilidades de desenvolvimento econômico. Os exemplos da diversidade cultural e modelos de tecnologias sociais em comunidades no Brasil e na Argentina apontam resultados efetivos. O diferencial dos exemplos se constitui no esforço para assegurar a identidade das comunidades e na abordagem da qualidade de vida em geração de renda.
\end{abstract}

Palavras-Chave: Diversidade cultural; tecnologias sociais; Brasil; Argentina; comunidades.

\footnotetext{
${ }^{1}$ Artigo apresentado no Simpósio Temático 04 - Políticas públicas, diversidade cultural e descolonização, durante o II Seminário Latino-Americano de Estudos em Cultura - SEMLACult em Foz do Iguaçu/PR, Brasil, 2018.

${ }^{2}$ Mestra e Doutoranda em Design; CEDTec - Centro de Estudos em Design e Tecnologia da Escola de Design da Universidade do Estado de Minas Gerais/UEMG; Cidade de Belo Horizonte/ Estado de Minas Gerais/Brasil; nadjamourao@gmail.com.
}

\footnotetext{
${ }^{3}$ PhD Gestão de Inovação Tecnológica/ECP França; Professora PPGD/UEMG; Coordenadora do CEDTec da UEMG; Cidade de Belo Horizonte/ Estado de Minas Gerais/Brasil; rcengler@uol.com

${ }^{4}$ Mestra em Design; CEDTec - Centro de Estudos em Design e Tecnologia da Escola de Design da Universidade do Estado de Minas Gerais/UEMG; Cidade de Belo Horizonte/ Estado de Minas Gerais/Brasil; anaceliadesign@gmail.com.
} 


\begin{abstract}
Resumen
En tiempos actuales, para combatir las constantes crisis que asolan a las naciones en todos los continentes, los patrones preestablecidos ya no responden a las cuestiones vivenciadas por la humanidad. Hay una búsqueda constante por nuevos modelos de gestión, en todas las áreas y sectores que demandan el desarrollo. En este estudio, se presentan dos estudios en comunidades en América Latina (Brasil y Argentina) que construyen experiencias participativas para incentivar la autogestión. Se cree que las tecnologías sociales, oriundas de acciones gubernamentales y no gubernamentales, casi siempre apoyadas en políticas públicas, pueden generar posibilidades emprendedoras sociales, reafirmando sus especificidades identitarias. Por el método del análisis bibliográfico se realiza una búsqueda de temáticas que posibiliten una cobertura de contenidos y ejemplos que atiendan a los objetivos de la investigación. El evento "Encuentro de los Pueblos del Gran Sertão Veredas" se realiza en Chapada Gaúcha, en Minas Gerais / Brasil. el evento reúne a personas de otros municipios, estados y países. En medio de los festejos, se realizan la comercialización de productos locales por medio de la moneda social - Veredas. En Argentina, en la periferia de la ciudad de Buenos Aires, la plataforma "Caminos da Favela" busca soluciones a problemas estructurales de las comunidades, que participan en la identificación de esos problemas y promueven la integración entre los miembros. Se percibe que las tecnologías sociales además de solucionar problemas puntuales reafirman la cultura y la identidad de las comunidades gestoras. Son caminos recorridos que se transforman en factores diferenciales para posibilidades de desarrollo económico. Los ejemplos de la diversidad cultural y modelos de tecnologías sociales en comunidades en Brasil y Argentina apuntan resultados efectivos. El diferencial de los ejemplos se constituye en el esfuerzo para asegurar la identidad de las comunidades y en el abordaje de la calidad de vida en generación de ingresos.
\end{abstract}

Palabras clave: Diversidad cultural; tecnologías sociales; Brasil; Argentina; comunidades.

\begin{abstract}
In current times, in order to combat the constant crises that plague nations on all continents, the pre-established patterns no longer address the issues experienced by mankind. There is a constant search for new management models in all areas and sectors that demand development. In this study, two studies are presented in communities in Latin America (Brazil and Argentina) that construct participatory experiences to encourage self-management. It is believed that social technologies, derived from governmental and non-governmental actions, almost always supported by public policies, can generate social entrepreneurial possibilities, reaffirming their identity specificities. By the method of bibliographical analysis a search of thematic is made that allows a range of contents and examples that meet the objectives of the research. The event "Meeting of the Peoples of Grande Sertão Veredas" is held in Chapada Gaúcha, in Minas Gerais / Brazil. event that brings together people from other cities, states and countries. In the midst of the festivities, local products are traded through the social currency - Veredas. In Argentina, on the outskirts of the city of Buenos Aires, the "Caminhos da Favela" platform seeks solutions to the structural problems of communities that participate in the identification of these problems and promote integration among members. It is noticed that social technologies besides solving specific problems reaffirm the culture and the identity of the management communities. It is the paths that have become differential factors for economic development possibilities. The examples of cultural diversity and models of social technologies in communities in Brazil and Argentina point to effective results. The differential of the examples is the effort to assure the identity of the communities and to approach the quality of life in income generation.
\end{abstract}

Keywords: Cultural diversity; social technologies; Brazil; Argentina; communities.

\title{
1. Introdução
}

As comunidades de periferia, em regiões de difícil acesso ou distanciadas de centros urbanos, sofrem consequências pela hibridez da pobreza estabelecida entre elas. A carência do poder aquisitivo representa um atraso no desenvolvimento econômico, acentuada pelas 
condições topográficas de cidades e povoados isolados. Canclini (2004) relata que as condições de baixa renda de uma comunidade e a desigualdade social merecem atenção específica dos governantes.

Considera-se que fatores econômicos, políticos, impactos ambientais entre outros, sejam responsáveis pela crise mundial. O Dicionário do Aurélio refere-se ao substantivo "crise", como uma mudança brusca ou uma alteração importante no desenvolvimento de um evento ou acontecimento. Essas alterações podem ser físicas ou simbólicas, de conjuntura ou momento perigoso, difícil ou decisivo, e inclusive por situações de perdas ou escassez.

Dessa forma, em tempos atuais, para combater as constantes crises que assolam as nações em todos os continentes, os padrões pré-estabelecidos não mais atendem as questões superadas pela humanidade. Há uma busca constante por novos modelos de gestão, em todas as áreas e setores que demandam o desenvolvimento. Contudo, mantêm o sistema capitalista enraizado nas organizações sociais e nos seus territórios (TEIXEIRA; SOUZA, 1995).

O conceito de desenvolvimento sustentável inclui o pensar globalmente e agir localmente como uma prerrogativa para melhorar a qualidade de vida humana no planeta, conforme Comissão de Brundtlant (WCED, 1987). Esse conceito advém da realidade, mas arrasta por décadas os impasses e decisões em cúpulas de líderes mundiais. Assim, comunidades afetadas pela escassez buscam modelos de desenvolvimento sistêmico, utilizando o compartilhamento sociocultural dos recursos angariados da diversidade cultural.

Com o intuito de preservar a riqueza da diversidade cultural dos países, a Organização das Nações Unidas para a Educação, a Ciência e a Cultura - UNESCO, em 2002, instituiu a "Declaração Universal sobre a Diversidade Cultural". Este primeiro instrumento tem o objetivo de promover e proteger a diversidade cultural e o diálogo intercultural entre as nações. Ele reconhece as múltiplas culturas como uma "herança comum da humanidade".

A diversidade cultural é um conceito que representa em vários aspectos, as diferentes culturas como a linguagem, as tradições, a culinária, a religião, os costumes, o modelo de organização familiar, a política, entre outras características próprias de um grupo de seres humanos que habitam um determinado território (REYGADAS, 2002).

As tecnologias sociais, na atualidade, se apresentam como alternativas para solução de problemas estruturais da sociedade, simples, de baixo custo, replicáveis e frequentemente apoiadas por programas governamentais. Para sua propagação, se fundamentam na relevância pela participação de pessoas das comunidades que as desenvolvem e na sustentabilidade nas soluções apresentadas. Encontram-se possibilidades efetivas de soluções para problemas nas áreas da educação, inclusão social, meio ambiente, energia, alimentação, habitação, saúde, 
trabalho, entre outros, por meio das tecnologias sociais, de acordo com Mourão (2017) apud Instituto de Tecnologia Social (2004).

Os procedimentos e técnicas aplicados em tecnologias sociais se associam aos modelos de organização coletiva, que produzam resultados positivos para a inclusão social e melhoria da qualidade de vida. É necessário as atividades implementadas pelas tecnologias sociais permitam o monitoramento e avaliação de objetivos, incentivando o desenvolvimento sustentável (MOURÃO, 2017).

Em culturas diferentes, em função das características locais, são aplicadas tecnologias sociais que possam atendam as questões das comunidades. No entanto, uma tecnologia social aplicada na África, para solução do transporte de água, por exemplo, poderá não ser servir em outra sociedade. As características do território são fundamentais, como a composição geográfica, cultura local, características sociais, ambientais, políticas, entre outras. Deste modo, os elementos materiais e imateriais podem determinar em uma sociedade, quais as ações se transformam em benefícios, que estimulam melhor qualidade de vida na região.

Nesse estudo, apresentam-se dois estudos em comunidades na América Latina (Brasil e Argentina) que constroem experiências participativas de autogestão. Acredita-se que as tecnologias sociais, oriundas de ações governamentais e não-governamentais, quase sempre apoiadas em políticas públicas, podem gerar possibilidades empreendedoras sociais, reafirmando suas especificidades identitárias.

Pelo método da analise bibliográfica realiza-se uma busca de temáticas que possibilitem uma abrangência de conteúdos e exemplos que atendam aos objetivos da pesquisa. De acordo com Tasca et al. (2010), a análise do contexto, a definição de um problema e das questões direcionadoras, dão início ao processo de pesquisa científica, motivando assim os pesquisadores a procurarem informações sobre determinada temática em bases bibliográficas.

Dessa forma, apresentam-se exemplos da diversidade cultural e modelos de tecnologias sociais em comunidades no Brasil e na Argentina que desenvolvem habilidades para a autogestão. O diferencial dos exemplos se constitui no esforço para assegurar a identidade das comunidades e na abordagem da qualidade de vida em geração de renda.

\section{Encontro dos Povos em Chapada Gaucha}

O município de Chapada Gaúcha, emancipado a partir de 1995, está bem localizada geopolítica e mercadológica no norte do Estado de Minas Gerais/Brasil. É uma cidade nova, 
planejada e plana, cuja população mistura os sertanejos e Gaúchos - colonizadores desde os anos de 1970, oriundos da migração do Estado do Rio Grande do Sul (pelo projeto PADSA Projeto de Assentamento Dirigido ao Distrito de Serra das Araras), atualmente com uma população de 10.792 habitantes, numa área de 3.214,698 km² (IBGE, 2010).

No entorno do município estão localizadas duas Unidades de Conservação Estaduais. Há também o Parque Nacional Grande Sertão Veredas, que é uma das atrações turísticas na região, situada na divisa dos estados de Minas Gerais e da Bahia. Outro destaque é o Parque Estadual Serra das Araras e Reserva Estadual de Desenvolvimento Sustentável Veredas do Acari. É uma região rica em biodiversidade, mas isolada e com carências para o desenvolvimento econômico.

A partir do ano de 2002, a Prefeitura Municipal de Chapada Gaúcha, com o apoio de instituições parceiras do município, realiza o evento "Encontro dos Povos do Grande Sertão Veredas". O evento reúne pessoas de outros municípios, estados e países. Em meio aos festejos, são encontrados pessoas visitantes, pesquisadores e turistas.

Durante todos os dias do evento, os produtos artesanais das comunidades do Vale do Urucuia são destaques e geram resultados econômicos para as comunidades. Ocorrem seminários e debates que tratam de problemas e soluções em tecnologias sociais como as barraginhas para retenção de água de chuva, adequação em plantio e coleta dos frutos do cerrado e sistema de preservação da natureza. Também são apresentadas diversas atividades culturais, educacionais, ambientais.

O Encontro dos Povos do Grande Sertão Veredas a participação das pessoas das comunidades envolvidas e também turistas de todo Brasil, pela riqueza cultural e manifestações exibidas durante os dias do evento (Figura 1).

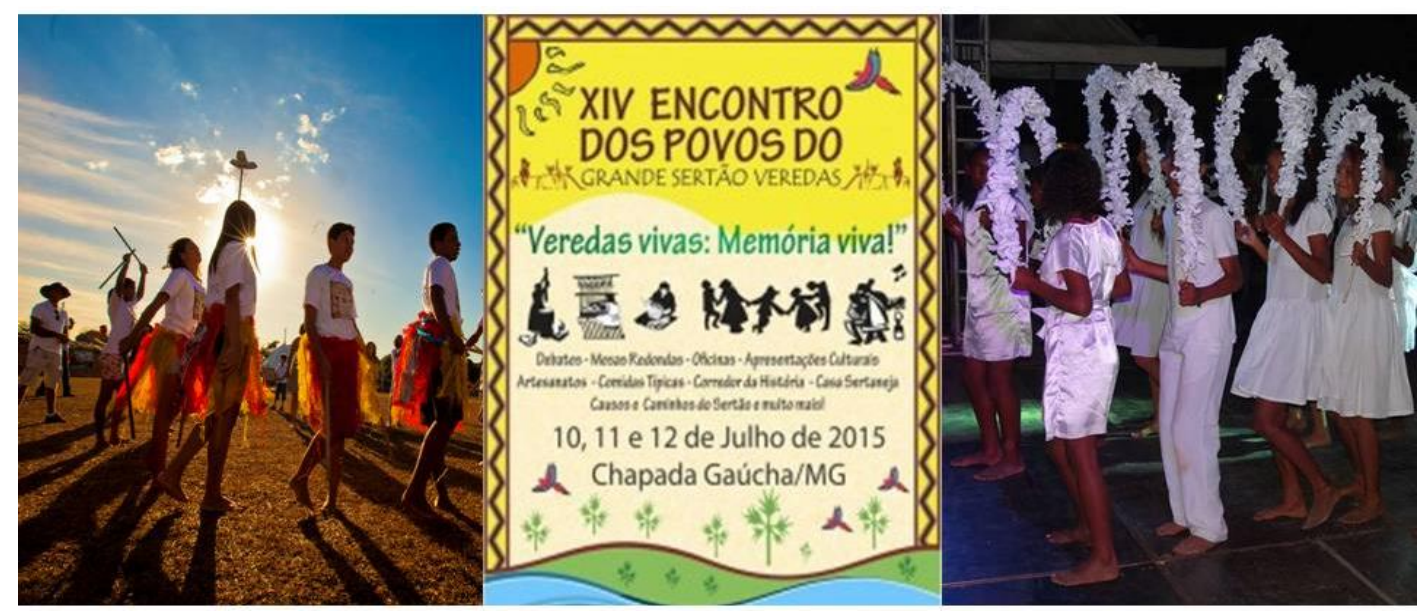

Figura 1 - Encontro dos Povos do Grande Sertão Veredas.

Fonte: Acervo da pesquisa, 2018. 
Apresentações folclóricas e danças de varias origens acontecem no palco, no pátio central da festa e entre as barracas de artesanato e de comidas tradicionais. A comercialização dos produtores locais utiliza a moeda social "Veredas" - certificada como Tecnologia social pela Fundação Banco do Brasil, para manter os recursos econômicos na região.

As moedas sociais, em diferentes regiões do mundo, apontam soluções para a economia local, a partir da iniciativa de Muhammad Yunus, na Índia, em 1976. No Brasil, foi o Banco Palmas, o primeiro banco social, criado em 1998, pela Associação dos Moradores do Conjunto Palmeira, bairro de Fortaleza/ Ceará. Para facilitar as transações das moedas sociais, foi criado o aplicativo E-dinheiro, que pode ser acessado em suportes digitais.

As moedas sociais são facilitadoras da o empreendedorismo social. O conceito de empreendedorismo social nasce em contexto americano e que não obstante as divergências entre as escolas que se debruçam sobre o fenômeno, fruto dos contextos sociais, políticos e econômicos diferenciados, todas contribuíram quer para o crescimento do interesse pelo tema quer para a consensualização da necessidade de encontrar formas inovadoras no uso de métodos de negócio adequados à resolução de problemas sociais (PARENTE et al., 2011).

A primeira moeda social no Estado de Minas Gerais foi a Veredas (Figura 2), comercializada desde 2009. Foi batizada assim em alusão ao romance "Grande Sertão: Veredas”, de Guimarães Rosa (1908-1967), nascido em Cordisburgo, região do Vale do Urucuia (MOURÃO, 2018).

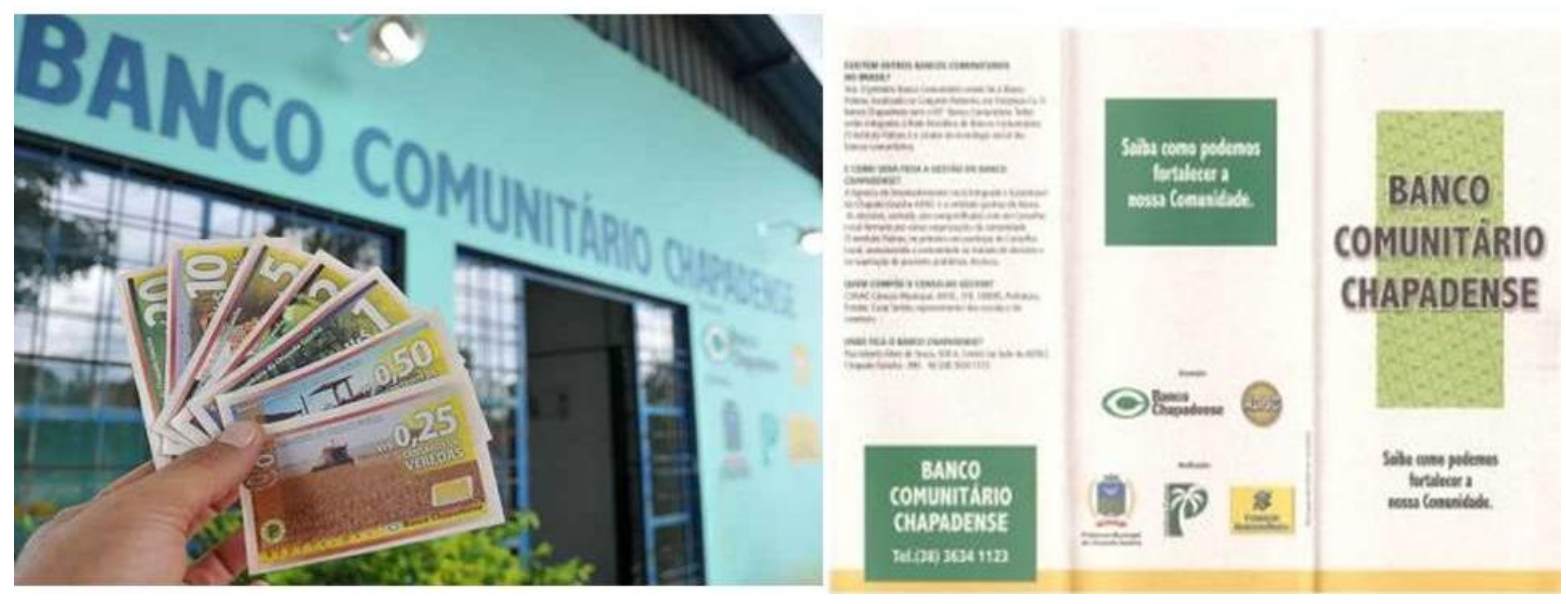

Figura 2 - Moeda e Banco Veredas de Chapada Gaúcha. Fonte: Acervo da pesquisa, 2018.

A moeda Veredas circula no município no lugar do dinheiro convencional. Foi criado pela comunidade Chapadense com o apoio da FBB e o instituto Banco Palmas. Tem como 
objetivo o desenvolvimento da comunidade pelo fortalecimento das redes locais, mantendo a riqueza do município dentro do município (MOURÃO, 2018).

A diversidade da cultura entre os povos ribeirinhos, sertanejos, chapadeiros, quilombolas e muitos outros, é observada durante as apresentações dos grupos artísticos durante o evento. Esses povos aproveitam o evento para divulgar e preservar suas músicas, danças, comidas, entre outras manifestações da cultural dos povos que convivem na região.

\section{Caminhos da Favela (Caminos de la Villa - Argentina)}

Desenvolvido pela Asociación Civil por la Igualdad y la Justicia da Argentina, o projeto Caminhos da Favela busca promover a integração das comunidades segregadas socioespacialmente de Buenos Aires através do acompanhamento de obras públicas desenvolvidas em favelas, oportunizando o empoderamento dessas comunidades para garantir seu direito de acesso a habitação decente. Isso porque em Buenos Aires vivem pouco mais de 2,8 milhões de pessoas das quais 275.000 vivem em mais 20 favelas e assentamentos. As condições dessas favelas são críticas: a contaminação da água, ar e do solo; privadas de qualquer possibilidade de acesso a saneamento, coleta de lixo, transporte público, educação e oferta de saúde, conforme Banco de Tecnologias Sociais da Fundação Banco do Brasil.

"Caminhos da Favela" é um portal multimídia interativa (Figura 3) que torna visíveis as condições de vida de mais de 20 bairros segregados da Cidade de Buenos Aires, Argentina.

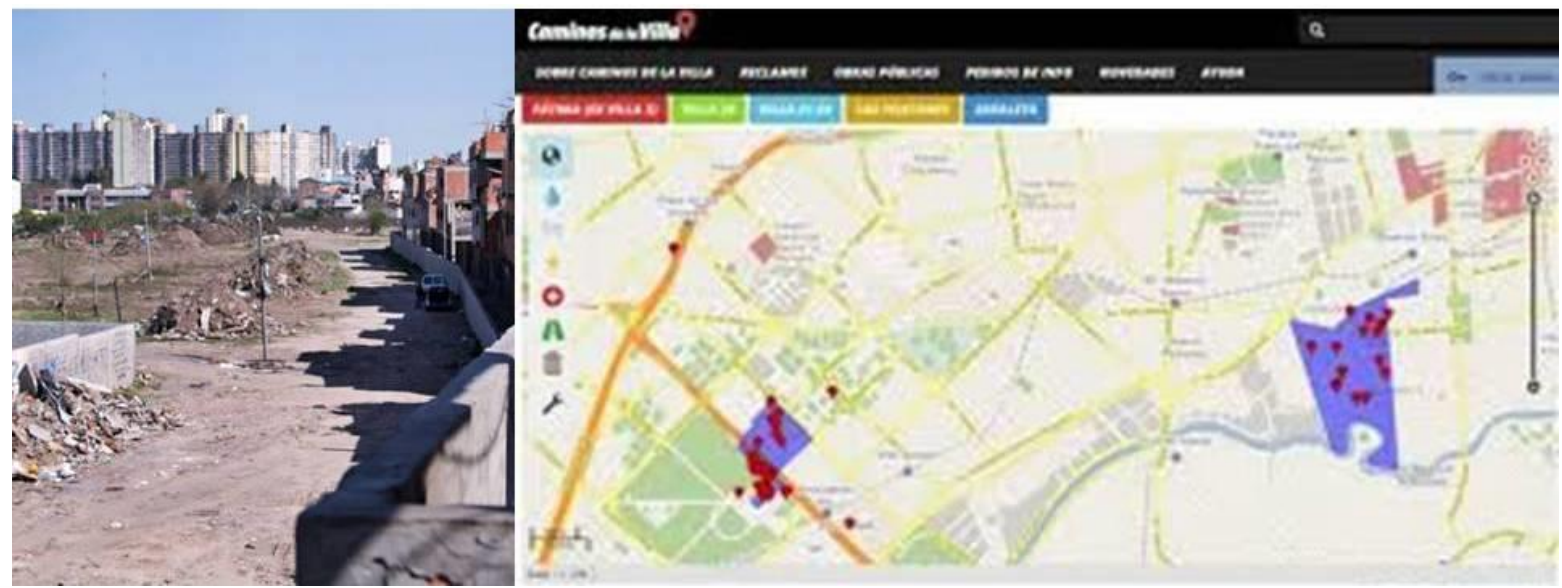

Figura 3 - Programa Caminhos da Favela, da cidade de Buenos Aires/Argentina.

Fonte: Banco de Tecnologias Sociais da FBB, 2018.

Esse portal virtual proporciona aos moradores desses bairros uma ferramenta para o diagnóstico da comunidade das diferentes prestações de serviços e de monitoramento e controle de obras públicas. A Fundação Banco do Brasil destaca que o mapeamento se faz de 
forma participativa, envolvendo atividades com a participação da comunidade. Cada morador tem a possibilidade de realizar o georreferenciamento de infraestruturas, equipamentos e marcos referenciais. Eles podem acrescentar valor nos espaços comuns, e desenvolver campanhas temáticas de identificação e visibilidade dos problemas a que estão expostos.

A participação dos moradores (usuários) tornou-se um fator estrutural em todo o processo de implementação da plataforma. Essa ação digital gera inclusive, a união da diversidade cultural, um fator de integração das favelas. Acredita-se que a plataforma Caminhos da Favela, como tecnologia social, possa facilitar a organização para melhorar a mobilidade, o comercio e a geração de renda nas favelas integradas.

O número de usuários registrados cresceu significativamente após as oficinas oferecidas em bairros, campanhas temáticas e a disseminação da plataforma em vários meios de comunicação e nas redes sociais. Até o momento de registro da plataforma no site da Fundação Banco do Brasil, em 2017, foram enviados 123 conjuntos de dados para a plataforma, composta por trabalhos realizados em diferentes bairros. Estima-se que 15.000 usuários já estejam colaborando na resolução de problemas locais (FBB, 2018).

Esse modelo é um processo evolutivo utilizado por muitas outras regiões urbanas no mundo com problemas semelhantes. No Rio de Janeiro, Brasil, os moradores do Complexo de Manguinhos colaboraram para a inclusão de sua comunidade no mapa do Rio, com o apoio do Instituto Pereira Passos (IPP). Eles mapearam escolas, creches, centros culturais, bares, lojas e passaram as informações para o aplicativo Mapa Participativo/RJ, conforme Lapagesse (2014). Essa é uma tecnologia social que além de contribuir para melhoria da qualidade de vida, gera possibilidades de conhecimento das diversas culturas existentes nos territórios.

\section{Análise dos resultados}

Dois estudos de caso da América Latina se constituem em experiências participativas que favorecem a solução de problemas locais, incentivando a autogestão da comunidade. Em cada exemplo encontra-se o potencial da cultura local com base para a resolução de problemas locais.

O domínio do conhecimento de cada grupo social (no caso da Comunidade de Chapada Gaúcha/ Brasil e de Buenos Aires/ Argentina) se transforma em fatores diferenciais para a geração de possibilidades socioeconômicas.

A moeda social Veredas (em Chapada Gaúcha) além de ser, por sua forma de apresentação, uma referencia ao contexto do Cerrado e das Veredas, exaltando o trabalho de 
Guimarães Rosa, é uma formadora de novas interpretações culturais. Ela se utiliza da cultura territorial como estratégia de valorização do seu patrimônio. No caso da plataforma Caminos De La Villa (Buenos Aires) verifica-se a expansão de registros físicos relevantes ao contexto da cultura local.

Percebe-se que as tecnologias sociais além de solucionarem problemas pontuais reafirmam a cultura e a identidade das comunidades gestoras. São caminhos percorridos que se transformam em fatores diferenciais para possibilidades de desenvolvimento econômico.

\section{Considerações Finais}

A diversidade cultural contribui para preservação da estrutura e dos valores de comunidades, que podem utilizar de eventos culturais ou mesmo de recursos digitais para solução de problemas locais.

Nessa análise, além da diversidade cultural, observa-se que as relações entre as pessoas e o espaço físico são complexas e multifacetadas, e ocorrem em diferentes escalas de influência.

Cada indivíduo possui as suas características pessoais e o ambiente incorpora os valores sociais e culturais de todos os que nele vivem. Assim, os exemplos apontam que as características locais são fatores que influenciam os resultados conforme o contexto histórico, étnico, territorial e sociocultural da comunidade.

São primários os estudos a cerca da importância da diversidade cultural na resolução de problemas sociais. Verifica-se a necessidade de novas pesquisas em geração de renda a partir das tecnologias sociais, apresentando a diversidade cultural como temática regional. Contudo, considera-se que a estratégia de participação das comunidades para resolução dos problemas locais é uma das possibilidades que se apresenta em modelos diversificados.

\section{Referências}

CANCLINI, N. G. Diferentes, desiguales y desconectados. Mapas de la interculturalidad. Barcelona: Gedisa, 2004.

DICIONÁRIO DO AURÉLIO. Significado de Crise. Disponível em: 〈https://dicionariodoaurelio.com/crise>. Acesso em: 10 mai. 2019.

FUNDAÇÃO BANCO DO BRASIL - FBB. Caminhos Da Favela (Caminos De La Villa Argentina). Postado em: Atualizada em: 16 mar. 2018. Disponível em: 
$<$ http://tecnologiasocial.fbb.org.br/tecnologiasocial/banco-de-tecnologiassociais/pesquisar-tecnologias/detalhar-tecnologia-741.htm>. Acesso em: 10 mai. 2019.

INSTITUTO BRASILEIRO DE GEOGRAFIA E ESTATISTICA - IBGE. Censo 2010. Disponível em: 〈https://censo2010.ibge.gov.br/resultados.html〉.Acesso em: 10 mai. 2019.

INSTITUTO DE TECNOLOGIA SOCIAL - ITS. Disponível em: <http://www.itsbrasil.org.br/conheca-o-its-brasil/conheca-o-its-brasil >. Acesso em: 10 mai. 2019.

LAPAGESSE, G. Com a ajuda de novo aplicativo, moradores põem favelas com UPPs no mapa do Rio Comunidade de Manguinhos é a primeira no Rio a participar do projeto. Postado em: 01 nov. 2014. Disponível em: <https://oglobo.globo.com/rio/com-ajuda-denovo-aplicativo-moradores-poem-favelas-com-upps-no-mapa-do-rio-14433152>. Acesso em: 10 mai. 2019.

MOURÃO, N. M. Apostila Tecnologia Social e design para todos. Belo Horizonte: CEDTec - Centro de Estudos em Design e Tecnologia da Escola de Design da Universidade do Estado de Minas Gerais, 2018.

MOURÃO, N. M. Tecnologias sociais e empreendimentos criativos na Região Metropolitana de Belo Horizonte/MG. Revista Brasileira do Desenvolvimento Regional, PPGDR/Universidade Regional de Blumenau, ISSN 2317-5443, 2017. DOI: <http://dx.doi.org/10.7867/2317-5443.2017v5n1p053-067>. Acesso em: 10 mai. 2019.

PARENTE, C.; COSTA, D.; SANTOS, M.; CHAVES, R. R. Empreendedorismo social: contributos teóricos para a sua definição. In: XIV Encontro Nacional de Sociologia Industrial, das Organizações e do Trabalho Emprego e coesão social: da crise de regulação à hegemonia da globalização. Lisboa, 26 e 27 de Maio de 2011. Disponível em: <file:///C:/Users/cdetec/Downloads/Parente-2011-EmpreendedorismoSocial.pdf>. Acesso em: 10 mai. 2019.

REYGADAS, L. Ensamblando culturas: diversidad y conflicto en la globalización de la industria. Barcelona: Gedisa, 2002.

TASCA, J. E. et al. An approach for selecting a theoretical framework for the evaluation of training programs. Journal of European Industrial Training, v. 34, n. 7, p. 631- 655, 2010. <http://dx.doi.org/10.1108/03090591011070761>. Acesso em: 10 mai. 2019.

TEIXEIRA, D. L. P.; SOUZA, M. C. A. F. de. Organização do processo de trabalho na evolução do capitalismo. Revista de Administração de Empresas, vol.25 nº. 4, São Paulo Oct./Dec. 1985. Disponível em: $<$ http://dx.doi.org/10.1590/S003475901985000400007>. Acesso em: 10 mai. 2019.

UNESCO. Declaração Universal sobre a Diversidade Cultural. CLT.2002/WS/9, 2002. Disponível em: 〈http://unesdoc.unesco.org/images/0012/001271/127160por.pdf>. Acesso em: 10 mai. 2019.

WCED - WORLD COMMISSION ON ENVIRONMENT AND DEVELOPMENT (Gro Harlem Brundtland, Chair). Our Common Future. Oxford University Press, Oxford, 1987. 